\title{
NUMERICAL SIMULATION OF MINOR LOSSES IN OSCILLATING FLOW
}

\author{
S. Mukhopadhyay* and B. J. Boersma \\ Process and Energy Department, Delft University of Technology, Delft, The Netherlands. \\ *Corresponding author's e-mail: s.mukhopadhyay@tudelft.nl
}

Keywords: oscillating flow, diffuser, minor loss, RANS

\section{Introduction}

Thermoacoustic devices operate on conversion of heat into acoustic energy and vice versa for use in heat engines or refrigerators [2]. Normally the conversion between thermal and acoustic energies is realised by oscillating gas flow. The main advantages are lack of moving mechanical parts and use of non polluting inert gases as working medium. The present efficiency levels are as high as $49 \%$ of the Carnot efficiency [3] but the efficiency levels need further improvement to take them to commercial scales. Significant improvement can be achieved by reducing losses in pipe bends, expansions or entrances (other than in a straight passage) which are generally termed as minor losses. Minor losses in oscillating flows can dissipate significant acoustic power in thermoacoustic heat pumps and refrigerators [2]. Numerical simulation of minor losses can help in improving the design of thermoacoustic devices. In this work, we investigate performance of Reynolds-Average Navier-Stokes (RANS) method in capturing minor losses in incompressible oscillating flow by comparing with available experimental data.

\section{Problem Formulation and Numerics}

Air flow in a 2-D diffuser section with $30^{\circ}$ angle is chosen from the experimental studies of oscillating flows in 2-D diffuser [1]. Oscillation amplitude and a measure of driving frequency are required to define oscillating flow in a channel. The amplitude can be described by Reynolds number defined as- $R e_{\delta}=\frac{u_{\max } \delta}{v}$ where $u_{\max }$ is the maximum velocity through the cycle in the channel, $v$ is the kinematic viscosity and $\delta=\sqrt{v / \pi f}, f$ is the oscillation frequency.
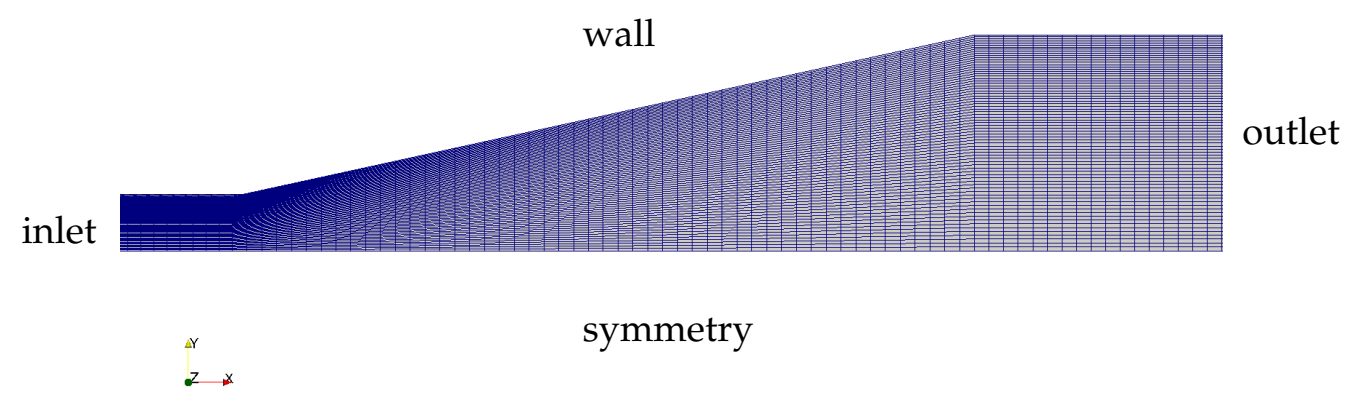

Figure 1: Schematic representation of the computational domain, mesh and boundary conditions.

The Computational Fluid Dynamics (CFD) code Open Source Field Operation and Manipulation (OpenFOAM) based on the finite volume method (FVM) is used for the numerical simulations. The standard high Reynolds number $k-\epsilon$ model along with wall function is applied as it is most commonly used in industry. The OpenFOAM transient solver pimpleFoam (based on merged PISO-SIMPLE algorithm) for incompressible flow is used with second order dis- 
cretisation schemes. Only half of the domain is simulated taking advantage of symmetry and boundary conditions are shown in Figure 1. At inlet a sinusoidal velocity profile is prescribed. To capture the unsteady flow physics and ensure convergence small time steps are used, automatically adjusted by the solver to maintain Courant number below 0.9. Flows with $R e_{\delta}=740$ are investigated for different dimensionless displacement amplitude, $L_{0} / h$ as given in Table 1.

Table 1: Oscillation frequencies simulated at $R e_{\delta}=740$ for $30^{\circ}$ diffuser

\begin{tabular}{lccccc}
\hline$L_{0} / h$ & 25 & 28 & 31 & 35 & 40 \\
\hline$f(\mathrm{~Hz})$ & 20.41 & 16.27 & 13.27 & 10.41 & 7.97 \\
$u_{\max }(\mathrm{m} / \mathrm{s})$ & 26.67 & 23.81 & 21.51 & 19.05 & 16.67 \\
\hline
\end{tabular}

\section{Results and Discussion}

The results for sum of minor losses $\left(K_{B}-K_{S}\right)$ and normalized acoustic power dissipation $\left(\dot{E} / \rho A u^{3}\right)$ computed like [1] are presented as function of $L_{0} / h$ in Figure 2. The results are in line with experimental findings with the deviations more pronounced at low oscillating frequencies. It is found that minor losses and acoustic power dissipation grow with increasing displacement amplitude (or decreasing oscillating frequency). Thus standard high Reynolds number $k-\epsilon$ model is able to predict the trend but further investigation is required to improve quantitative prediction. Additional studies will be presented during the workshop.
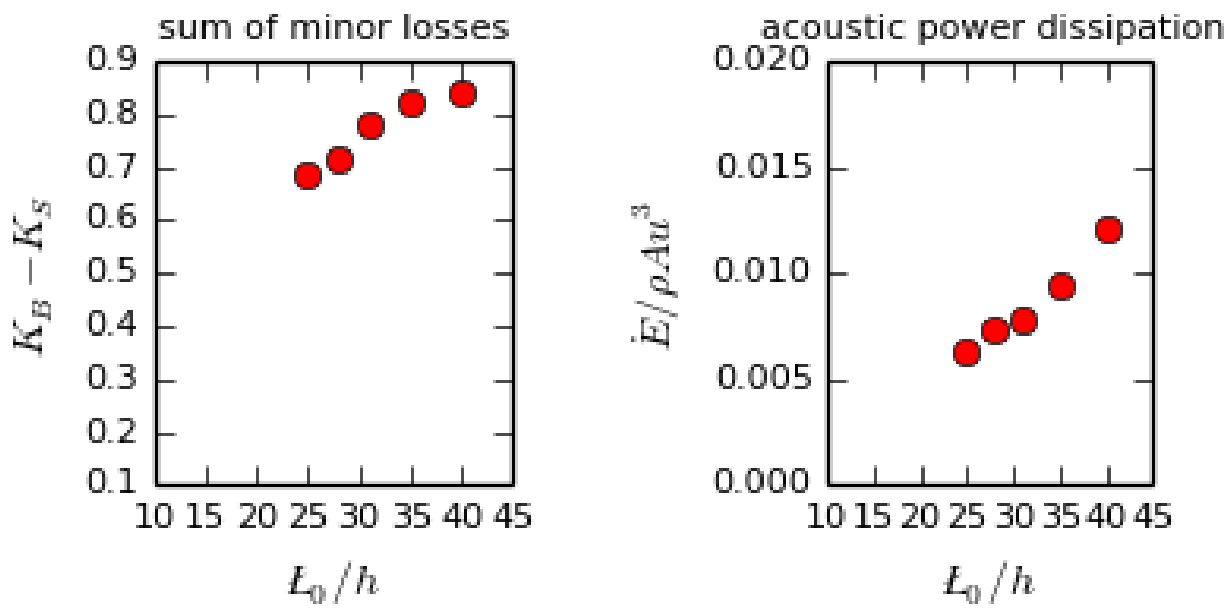

Figure 2: Minor losses $\left(K_{B}-K_{S}\right)$ and normalized acoustic power dissipation $\left(\dot{E} / \rho A u^{3}\right)$.

\section{Acknowledgements}

The authors are grateful to Energy research Centre of the Netherlands (ECN) for funding this research work.

\section{References}

[1] King, C. V. and Smith, B. L. “Oscillating flow in a 2-D diffuser". Experiments in Fluids, 51, (2011), 1577-1590.

[2] Swift, G. Thermoacoustics: A Unifying Perspective for Some Engines and Refrigerators. Acoustical Society of America (2002).

[3] Tijani, M. E. H. and Spoelstra, S. "A high performance thermoacoustic engine". Journal of Applied Physics, 110, 093519 